\title{
One-year survival and resource use after critical illness: impact of organ failure and residual organ dysfunction in a cohort study in Brazil
}

\author{
Otavio T. Ranzani ${ }^{1{ }^{*}}$, Fernando G. Zampieri ${ }^{1 \dagger}$, Bruno A. M. P. Besen ${ }^{1}$, Luciano C. P. Azevedo ${ }^{1,2}$ and Marcelo Park ${ }^{1,2}$
}

\begin{abstract}
Introduction: In this study, we evaluated the impacts of organ failure and residual dysfunction on 1-year survival and health care resource use using Intensive Care Unit (ICU) discharge as the starting point.

Methods: We conducted a historical cohort study, including all adult patients discharged alive after at least $72 \mathrm{~h}$ of ICU stay in a tertiary teaching hospital in Brazil. The starting point of follow-up was ICU discharge. Organ failure was defined as a value of 3 or 4 in its corresponding component of the Sequential Organ Failure Assessment score, and residual organ dysfunction was defined as a score of 1 or 2 . We fit a multivariate flexible Cox model to predict 1 -year survival.
\end{abstract}

Results: We analyzed 690 patients. Mortality at 1 year after discharge was $27 \%$. Using multivariate modeling, age, chronic obstructive pulmonary disease, cancer, organ dysfunctions and albumin at ICU discharge were the main determinants of 1-year survival. Age and organ failure were non-linearly associated with survival, and the impact of organ failure diminished over time. We conducted a subset analysis with 561 patients (81\%) discharged without organ failure within the previous $24 \mathrm{~h}$ of discharge, and the number of residual organs in dysfunction remained strongly associated with reduced 1-year survival. The use of health care resources among hospital survivors was substantial within 1 year: $40 \%$ of the patients were rehospitalized, $52 \%$ visited the emergency department, $90 \%$ were seen at the outpatient clinic, $14 \%$ attended rehabilitation outpatient services, $11 \%$ were followed by the psychological or psychiatric service and $7 \%$ used the day hospital facility. Use of health care resources up to 30 days after hospital discharge was associated with the number of organs in dysfunction at ICU discharge.

Conclusions: Organ failure was an important determinant of 1-year outcome of critically ill survivors. Nevertheless, the impact of organ failure tended to diminish over time. Resource use after critical illness was elevated among ICU survivors, and a targeted action is needed to deliver appropriate care and to reduce the late critical illness burden.

\section{Introduction}

The effects of critical illness on patients' lives do not cease after intensive care unit (ICU) discharge [1-9]. Patients who survive ICU stays have higher hospital and posthospital mortality than other patients and the general population $[2,10]$. Furthermore, ICU survivors are at risk of physical, cognitive and mental health consequences [11-13]. Post-ICU mortality seems to be greatest during

\footnotetext{
*Correspondence: otavioranzani@yahoo.com.br

${ }^{\dagger}$ Equal contributors

'Intensive Care Unit, Emergency Medicine Discipline, Hospital das Clínicas, University of São Paulo, Rua Dr. Enéas de Carvalho Aguiar, 255, 5th floor, Room 5023, São Paulo 05403-010, Brazil

Full list of author information is available at the end of the article
}

the first month after ICU discharge, suggesting that even hospital discharge is inaccurate for measuring the impact of critical illness on outcomes [1, 2, 14, 15]. Furthermore, increased health resource use by survivors of critical illness have been described recently in developed countries, representing a substantial component of resource use in the health care system [16-22].

The burden of organ failure, exacerbated inflammatory response and other events (e.g., endothelial activation, physiological derangements) that occur during the acute phase of critical illness are clearly associated with poor short-term outcomes, as captured by ICU and hospital mortality. In contrast, the extent to which these residual effects might influence long-term outcomes is uncertain 
[1, 14, 23-27]. The cumulative [25] and peak maximum organ failure [26] were associated with long-term mortality; however, in a large cohort of patients with septic shock, the impacts of these factors seemed to be strongest only early after admission [28]. The starting point for describing long-term outcomes is fundamental [1, 29, 30], and the majority of studies have usually started their follow-up periods at ICU admission, including ICU death as a long-term prognosis and perhaps attenuating the impact of organ failure on post-ICU outcomes $[1,26,29,30]$. In a further step, researchers in a recent study differentiated the determinants of short- and long-term survival after critical illness [1]. Considering the dynamic process of critical illness, this approach is fundamental for the prognostication and discussion of treatment plans, for research and for prevention from both the patient and health system perspectives $[1,26,31]$.

The time point of ICU discharge is an intermediate phase that represents an enormous gap in care, and it could be essential to long-term outcomes [8, 29]. Indeed, it is a rich moment for actions (e.g., early rehabilitation) to reduce early complications and to plan treatment goals before hospital discharge [7]. We therefore aimed to evaluate the impacts of organ failure and residual dysfunction on 1-year survival and health care resource use with ICU discharge as the starting point. We hypothesized that the impact of organ dysfunction on prognosis would be more pronounced during the first 90 days, with decreasing impact during the 1 year follow-up period. As secondary endpoints, we evaluated the impact of organ dysfunction on health care use at a public tertiary service in Brazil.

\section{Methods \\ Population}

We conducted a historical cohort study that included all patients admitted to a general adult ICU at a tertiary teaching hospital in São Paulo, Brazil, between January 2003 and December 2008. We analyzed patients who were discharged alive from the ICU following at least a 72-h stay. This criterion was chosen because these patients are prone to higher post-ICU mortality than other patients [32]. For patients with multiple ICU admissions, only the first admission was recorded.

\section{Health care system}

Our ICU is located in the Hospital das Clínicas, the largest health care complex in Latin America. At the time the study was conducted, it consisted of 7 specialized institutions with a total of 2,400 beds. There were also one rehabilitation hospital and one long-term acute care facility.

\section{Our intensive care unit}

Our unit is a mixed ICU with seven beds. This unit follows current standard of care practices, including sedation, nutritional, mechanical ventilation and hemodynamic monitoring protocols. An intensivist was available on site $24 \mathrm{~h}$ per day, 7 days per week (24/7). The staff consisted of one senior physician, one critical care fellow and three residents from the internal medicine program. At night, there was one senior physician and one resident. The health staff consisted of two nurses and three nursing assistants on a $24 / 7$ schedule, in addition to a respiratory therapist who was on a $12 / 7$ schedule [33]. The unit was a closed unit and received patients from the entire health care complex, although the majority of patients came from the Emergency Department and the medical wards. Patients who underwent cardiothoracic surgery, transplants and elective surgery (e.g., neurosurgical, vascular), as well as trauma and burn patients, are usually admitted to other specialized ICUs in the complex.

This study protocol followed the statements of the Declaration of Helsinki. The hospital system's institutional review board [Comissão para Análise de Projetos de Pesquisa (CAPPesq)] reviewed and approved this study (CAPPesq protocol number 107443). The requirement for written informed consent was waived because there was no intervention; we used only a database that guaranteed patient confidentiality.

\section{Data collection}

All of the data were recorded prospectively with a computerized physician order entry system. The admission data included patient age, reason for admission, physiological data and Acute Physiology and Chronic Health Evaluation II (APACHE II) score [34]. Organ dysfunction was measured daily using the Sequential Organ Failure Assessment (SOFA) score [35], which includes six types of organ dysfunction in a numeric chart. The daily SOFA score, discriminated by each system evaluated, was recorded. The neurological SOFA score was calculated based on the last evaluation available done when the patient was not sedated. For unique missing values (e.g., if serum bilirubin value was not available), we used the mean value calculated based on the preceding results and the results after the missing value [36]. Our unit uses the SOFA score daily in clinical rounds, and we provide monthly training sessions in its use. We observed only $2 \%$ unique missing values for SOFA scores in our study.

\section{Organ dysfunction definitions}

Total SOFA score was calculated as the sum of each organ score daily during the ICU stay of each patient. The maximum SOFA score was the highest score for the total SOFA during the ICU stay. Organ failure was 
defined as a value of 3 or 4 for its corresponding component of the SOFA score [8]. Residual organ dysfunction was defined as a score of 1 or $2[8,37]$.

\section{Follow-up}

The patients were followed for 1 year after their ICU discharges using the electronic system available at the Hospital das Clínicas. The electronic system is used for administrative purposes in all services at the health care complex, and every procedure, visit, laboratory examination, vital sign and other data gathered during hospitalization or outpatient visits is compulsorily recorded along with the date and a unique identifier. The health care complex has a policy of following its patients, and, as a general rule, at least one scheduled visit is required for the patient to be discharged. A proportion of these patients attended an intensive care follow-up clinic managed by our ICU that was active from 2008 to 2011.

\section{Outcomes}

The primary endpoint for this study was 1 year survival after ICU discharge. We also evaluated the burden of critical illness 1 year after hospital discharge at the health care complex, including visits to the Emergency Department; outpatient clinic visits; hospital readmissions; and other resource care use issues, such as consultation with psychiatric and psychological services, rehabilitation consultations (physiotherapy, nutrition and speech therapist visits), day hospital use and the number of complementary examinations performed.

\section{Statistical analysis}

Continuous data are presented as mean \pm standard deviation or as median and interquartile range, as appropriate. Categorical variables are shown as percentages. For categorical variables, Fisher's exact test or a $x^{2}$ test was used; for continuous variables, an unpaired $t$ test or the Mann-Whitney $U$ test was used if the data were normally or non-normally distributed, respectively.

To evaluate the pattern of SOFA score evolution over the ICU stay, we fit a generalized linear mixed model including the SOFA score at admission (D1) and sequentially on the third ICU day (D3) and at 72 (D - 3), $48(\mathrm{D}-2)$ and $24(\mathrm{D}-1)$ hours before ICU discharge, accounting for repeated measures correlation. To overcome overlapping of measurements for patients who stayed less than 6 days in the ICU, we maximized the time points for each patient, choosing the D1, D - 2 and $\mathrm{D}-1$ points for patients who stayed in the ICU for 3 days and D1, D - 3, D - 2 and D - 1 for patients who stayed in the ICU for 4 or 5 days. We conducted a sensitivity analysis defined post hoc, including only patients who stayed in the ICU for at least 6 days.
The Kaplan-Meier method was used to plot crude survival curves according to the number of organ dysfunctions and was compared using the log-rank test. We present survival and health care use with the same figures, as previously described by Prescott et al. [20]. In the Fig. 1, we plot a survival curve for mortality and a cumulative curve for hospital readmission.

The modeling of long-term outcomes is affected by the statistical model applied. Considering that critical illness has a dynamic effect on outcomes, following the inflammatory response, physiological disturbances and the complex scenarios involved in critical illness, the Cox proportional hazards $(\mathrm{PH})$ model, which is the most frequently used model, might not be accurate, because it relies on the assumption that the prognostic factors have constant hazard ratios over time and that continuous predictors present a linear function with the estimated risk. To address these issues, we fit flexible extensions of a Cox model that have been used with advantageous results $[14,28,38,39]$. Therefore, we used a flexible Cox model allowing for non-linear relationships between continuous predictors and survival and addressing timedependent covariates. The outcome was 1-year survival, and the main exposure was the SOFA score. To address potentially confounding factors, we ran a multivariate model, and variables were selected after bivariate analysis using a Cox $\mathrm{PH}$ model for the variables described in Table 1 . We selected variables associated with 1 year survival $(P<0.250)$. To choose the final multivariable model, we used the Akaike Information Criterion (AIC), looking for parsimonious models with the lowest AIC values [28] through a backward procedure. For each variable, we tested the $\mathrm{PH}$ assumption (through inspection of Schoenfeld residuals) and linearity between covariates and the log of hazard ratios. Variables that fulfilled both assumptions were entered as they would be into a traditional Cox $\mathrm{PH}$ model. We did not prespecify effect modifiers in our analysis. For continuous variables that did not fulfill the required assumptions, we applied a penalized smoothing spline function. The optimal degree of freedom for the spline was chosen by minimizing the AIC value [40]. Continuous variables were centered on the mean value. ICU length of stay was log-transformed because of its positively skewed distribution. To evaluate the discrimination of the final model, we used timedependent receiver operating characteristic (ROC) curves. Collinearity was assessed through the variance inflation factor (VIF), and a VIF value greater than 2.5 was considered to be suggestive of collinearity. To address losses to follow-up in the Cox regression, we censored patients lost to follow-up, assuming non-informative censoring. We observed missing data for only $2 \%$ of SOFA scores, for which we calculated mean values between previous and consecutive days around the missing values [36]. To 
Table 1 Characteristics of the patients included in the study

\begin{tabular}{|c|c|}
\hline Variables & All patients $(n=690)$ \\
\hline Age, yr, mean (SD) & $50.3 \pm 19$ \\
\hline Male sex, $n(\%)$ & 358 (52 \%) \\
\hline APACHE II score & $16[10-21]$ \\
\hline \multicolumn{2}{|l|}{ Type of admission, $n$ (\%) } \\
\hline Medical & $550(80 \%)$ \\
\hline Surgical & $140(20 \%)$ \\
\hline \multicolumn{2}{|l|}{ Comorbidities } \\
\hline Number of comorbidities, $n[\mathrm{IQR}]$ & $1[0-2]$ \\
\hline Hypertension, $n$ (\%) & 346 (50 \%) \\
\hline Diabetes, $n(\%)$ & 149 (22 \%) \\
\hline COPD, $n(\%)$ & $59(9 \%)$ \\
\hline Heart failure, $n(\%)$ & $110(16 \%)$ \\
\hline Chronic kidney disease, $n$ (\%) & $113(16 \%)$ \\
\hline AIDS, $n(\%)$ & 18 (3 \%) \\
\hline Cancer, $n(\%)$ & $71(10 \%)$ \\
\hline \multicolumn{2}{|l|}{ Reason for admission, $n(\%)$} \\
\hline Acute respiratory failure & 220 (32 \%) \\
\hline Shock & 125 (18 \%) \\
\hline Septic shock & $111(16 \%)$ \\
\hline CNS disorder & 78 (11\%) \\
\hline Monitoring & $73(10 \%)$ \\
\hline Post-operative period & 48 (7 \%) \\
\hline Gastrointestinal diseases & $50(7 \%)$ \\
\hline Electrolyte disturbances & $25(4 \%)$ \\
\hline Acute kidney injury & $23(3 \%)$ \\
\hline Trauma & $22(3 \%)$ \\
\hline \multicolumn{2}{|l|}{ Support during ICU stay, n (\%) } \\
\hline Mechanical ventilation & $510(74 \%)$ \\
\hline Renal replacement therapy & $102(15 \%)$ \\
\hline Vasopressors & $281(41 \%)$ \\
\hline \multicolumn{2}{|l|}{ SOFA score, median [IQR] } \\
\hline At admission & $4[2-7]$ \\
\hline Maximum & $6[3-9]$ \\
\hline At ICU discharge & $2[1-3]$ \\
\hline \multicolumn{2}{|l|}{ At least one organ failure, ${ }^{a} n(\%)$} \\
\hline At admission & $360(52 \%)$ \\
\hline Maximum & $428(62 \%)$ \\
\hline At ICU discharge & $129(19 \%)$ \\
\hline \multicolumn{2}{|l|}{ ICU length of stay, days } \\
\hline Mean \pm SD & $10 \pm 9$ \\
\hline Median [IQR] & $7[4-11]$ \\
\hline Albumin at discharge $(\mathrm{g} / \mathrm{L})$ & $27[23-31]$ \\
\hline
\end{tabular}

APACHE II Acute Physiology and Chronic Health Evaluation II, CNS Central nervous system, COPD chronic obstructive pulmonary disease, ICU Intensive
Care Unit, IQR interquartile range, SD standard deviation, SOFA Sequential Organ Failure Assessment

${ }^{a}$ Organ failure was defined as a value of 3 or 4 in a corresponding component of the SOFA score

estimate the hazard ratio over time, we used the approach suggested by Harrell [41], repeatedly reestimating the Cox regression model within time intervals. The log hazard ratios were plotted against the mean failure or censoring time within the interval [41]. We conducted a prespecified sensitivity analysis for patients without organ failure at ICU discharge. We employed the same analytical strategy reported above to evaluate the impact of residual organ dysfunction on 1-year survival.

Health care burden was assessed until the first year after ICU discharge. For the analysis, we conducted a prespecified sensitivity analysis by separating the associated burden, which occurred within the first 30 days after hospital discharge [42, 43].

All of the analyses were performed with $\mathrm{R}$ software, version 3.0.2. A $P$ value of 0.05 was considered to be significant for all comparisons.

\section{Results}

\section{Baseline characteristics}

Of the 1462 patients admitted to the ICU during the study period, 690 patients were discharged alive after a stay of at least $72 \mathrm{~h}$ and were therefore included in the present analysis (Additional file 1: Figure S1). Demographic data, reasons for admission, illness severity and other characteristics of the study population are described in Table 1.

\section{Organ failure and 1-year survival}

Four hundred twenty-eight patients (62\%) experienced at least one organ failure during their ICU stay. The time to present the greatest number of organ failures was less than $24 \mathrm{~h}$ for 284 patients $(65 \%)$. Ninety percent of our study sample demonstrated their greatest burden of organ dysfunction within the first 5 days of ICU admission.

Fifty patients (7\%) were lost to follow-up at 1 year. The general characteristics of the patients who were lost to follow-up were comparable to those of the general population (Additional file 1: Table S1). ICU mortality was $26 \%$. Following ICU discharge, hospital mortality for the index hospitalization was $18 \%$ (Table 3). For those patients discharged from the hospital, 1-year mortality was $9 \%$, totaling $27 \%$ of 1-year mortality for patients discharged alive after a 72-h ICU stay.

The survival curve is shown in Fig. 1. Compared with other patients, patients alive after 1 year had lower SOFA values at ICU admission, on the third day after admission, $72 \mathrm{~h}$ before discharge, $48 \mathrm{~h}$ before discharge and at discharge (Additional file 1: Figure S2A). Similar 


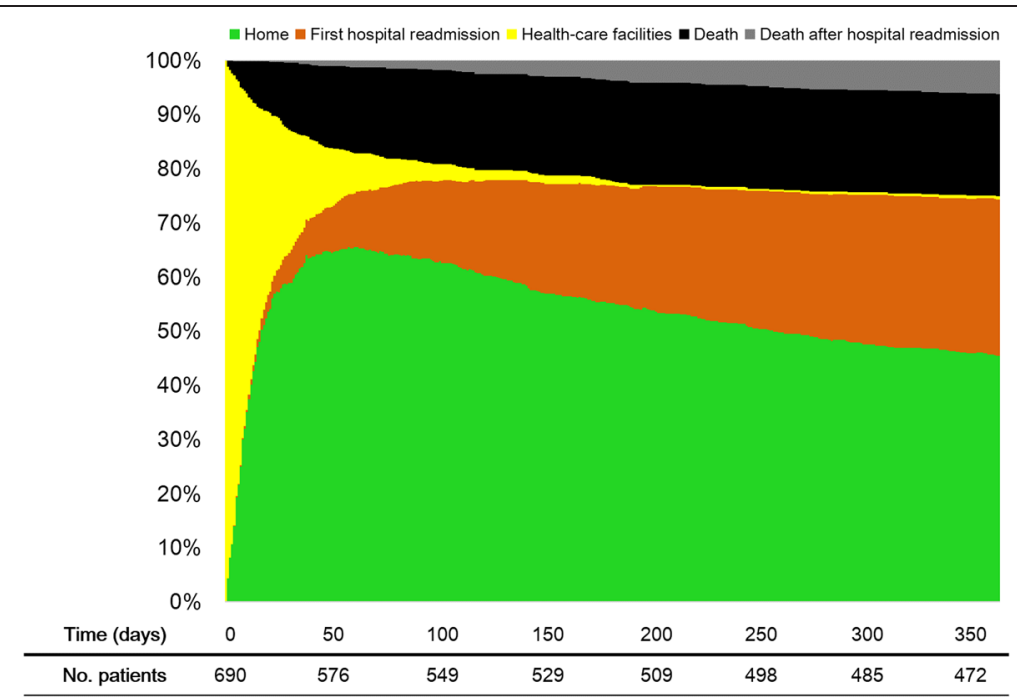

Fig. 1 Health care use and survival for 690 patients at 1 year after Intensive Care Unit discharge. Hospital readmission was considered to be major resource use, and, after the first rehospitalization, the patient was truncated in this category; otherwise, the patient was put into the death category if the patient died

results were found for patients who stayed at least 6 days in the ICU (Additional file 1: Figure S2B). The maximum SOFA score $\{6.0[95 \%$ confidence interval $(\mathrm{CI})$, 5.7-6.4] vs. 8.3 [95 \% CI, 7.6-8.9]; mean difference, -2.2 [95\% CI, -2.7 to -1.5$] ; P<0.001\}$ and mean SOFA score (3.2 [95 \% CI, 3.0-3.4] vs. 4.7 [95 \% CI, 4.3-5.1]; mean difference, -1.5 [95\% CI, -1.9 to -1.1$] ; P<0.001$ ) also differed between survivors and non-survivors.

Increasing numbers of organ failures had an additive negative impact on 1 -year survival $(P<0.001$ by log-rank test) (Additional file 1: Figure S3). Variables selected to be included in the Cox model were age, sex, arterial hypertension, chronic obstructive pulmonary disease, heart failure, chronic kidney disease, cancer, APACHE II score, medical admission, shock as a cause of admission, sepsis diagnosis, ICU length of stay, serum albumin values at ICU discharge and maximum SOFA score. APACHE II score, age and maximum SOFA score did not fulfill the $\mathrm{Cox} \mathrm{PH}$ criteria and therefore required the use of splines.
The results of the final model based on the AIC are shown in Table 2 and Figs. 2 and 3. The model had good discrimination, maintaining the area under the ROC curve at nearly 0.80 over time (Additional file 1: Figure S4).

The non-linear and time-varying effects of age and maximum SOFA score are shown in Figs. 2 and 3. The impact of organ failures, assessed by maximum SOFA score, tended to decrease over time.

At $24 \mathrm{~h}$ before ICU discharge, only 129 patients (19\%) had any organ failure (at least one SOFA score $\geq 3$ in each domain). We therefore conducted a subset analysis using the remaining 561 patients $(81 \%)$ discharged without organ failure over the previous $24 \mathrm{~h}$. Three hundred nine (55\%) of these patients had at least one organ failure during their ICU stay. At ICU discharge, 78 \% (435 of 561) had at least one residual organ dysfunction [median, 1 (IQR, 1-2) systems with residual organ dysfunction]. After modeling using the same approach as described above, the number of residual organ dysfunctions remained

Table 2 Multivariate analyses using a flexible Cox model for variables affecting 1-year survival after ICU discharge

\begin{tabular}{|c|c|c|c|c|}
\hline \multirow[b]{2}{*}{ Variable } & \multicolumn{2}{|c|}{ All patients $(n=690)$} & \multicolumn{2}{|c|}{ Patients without organ failure at ICU discharge $(n=561)$} \\
\hline & HR $(95 \% \mathrm{Cl})$ & $P$ value & $\mathrm{HR}(95 \% \mathrm{Cl})$ & $P$ value \\
\hline Age & Fig. $2 \mathrm{~A}$ & $<0.001$ & Additional file 1: Figure S6a & $<0.001$ \\
\hline COPD & $1.79(1.15-2.79)$ & 0.010 & Not included ${ }^{a}$ & - \\
\hline Cancer & $1.85(1.25-2.75)$ & 0.002 & $1.89(1.18-3.01)$ & 0.008 \\
\hline SOFA score & Fig. $3 A^{b}$ & $<0.001$ & Additional file 1: Figure $S 6 b^{c}$ & $<0.001$ \\
\hline Albumin at discharge ${ }^{d}$ & $0.96(0.94-0.99)$ & 0.005 & $0.96(0.93-0.99)$ & 0.010 \\
\hline
\end{tabular}

Cl confidence interval, COPD chronic obstructive pulmonary disease, HR hazard ratio, SOFA Sequential Organ Failure Assessment

${ }^{a}$ COPD was not retained in the final model in the sensitivity analysis, with 561 patients discharged without organ failure

${ }^{\mathrm{b}}$ Refers to maximum SOFA score observed during ICU stay, entered as a continuous variable and modeled as a non-linear term

${ }^{c}$ Refers to SOFA score at ICU discharge, entered as a continuous variable and modeled as a non-linear term

${ }^{d}$ Change in hazard ratio estimated for one unit change in albumin level measured in $\mathrm{g} / \mathrm{L}$. Albumin was modeled as a linear term in the Cox model 


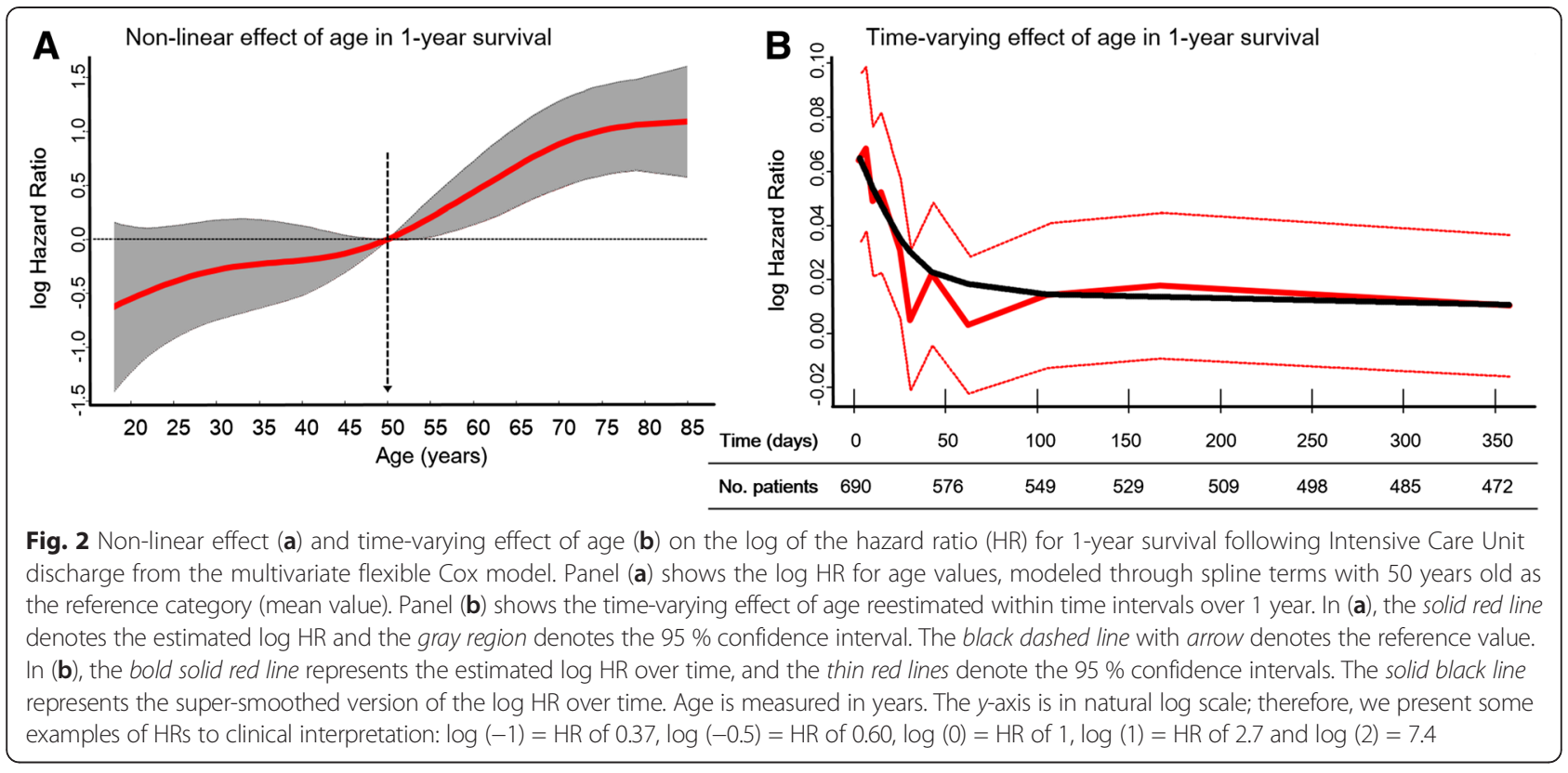

independently associated with reduced survival at 1 year following ICU discharge (Table 2). The impact of residual organ dysfunction was more pronounced when three or more residual organ dysfunctions were present at ICU discharge (Additional file 1: Figure S5).

\section{One-year health care use}

Of 690 patients discharged from the ICU, 565 patients (82\%) were discharged alive from the hospital after a median of 13 [6-28] days. Health care use in the year following discharge is shown in Table 3 and Fig. 1. After hospital discharge, $52 \%$ of the patients visited any emergency department of the hospital at least once, and $37 \%$ of patients were readmitted to the hospital after the index hospitalization, with $40 \%$ occurring after an emergency department visit. Furthermore, $90 \%$ of the patients attended outpatient clinic visits in several specialties during the year after ICU discharge. At 30 days postdischarge, contact with the health care system occurred for $32 \%$ because of readmission, $41 \%$ because of emergency department visits and $75 \%$ because of outpatient clinic visits (Additional file 1: Figure S7).

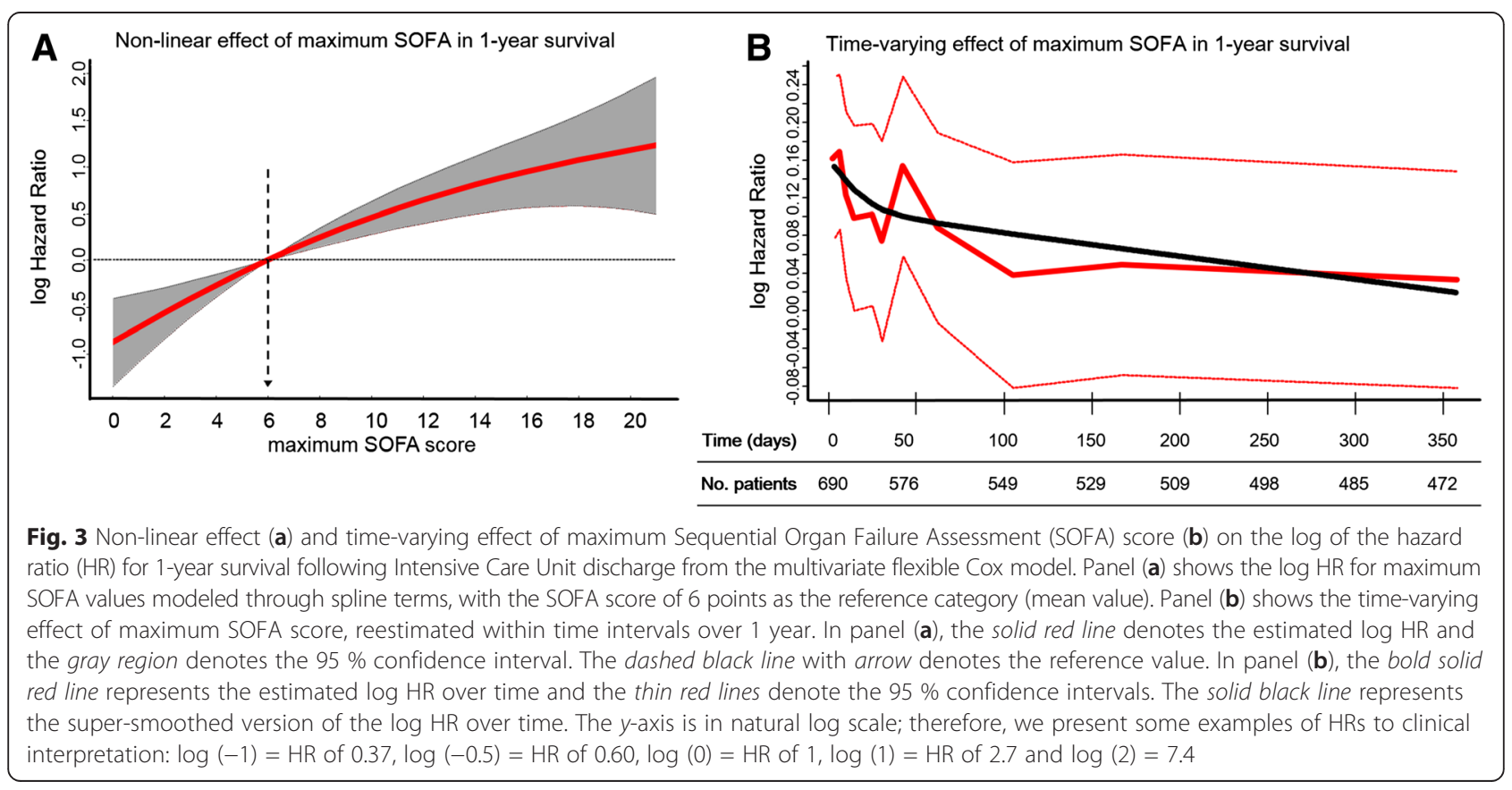


Table 3 Health care resource use in the year following ICU discharge

\begin{tabular}{|c|c|}
\hline Post-ICU facilities & Patients $(n=690)$ \\
\hline Ward & $530(77 \%)$ \\
\hline Step-down unit & $160(23 \%)$ \\
\hline Unplanned ICU admission & $121(18 \%)$ \\
\hline \multicolumn{2}{|l|}{ Hospital LOS after ICU discharge, days } \\
\hline Mean \pm SD & $23 \pm 28$ \\
\hline Median [IQR] (range) & $13[6-28](1-186)$ \\
\hline In-hospital mortality & $125(18 \%)$ \\
\hline Hospital discharge disposition & Patients $(n=565)$ \\
\hline Long-term acute care facility & $20(4 \%)$ \\
\hline Rehabilitation facility & $27(5 \%)$ \\
\hline Home & $518(91 \%)$ \\
\hline 1-yr health resource use & Patients $(n=534)^{a}$ \\
\hline Hospital readmissions & 199 (37 \%) \\
\hline Admission from emergency department ${ }^{b}$ & 79 (40 \%) \\
\hline \multicolumn{2}{|l|}{ Days to the first readmission } \\
\hline Mean \pm SD & $100 \pm 96$ \\
\hline Median [IQR] (range) & $69[17-154](1-360)$ \\
\hline \multicolumn{2}{|l|}{ Hospital LOS on the first readmission, } \\
\hline Mean \pm SD & $12 \pm 16$ \\
\hline Median [IQR] (range) & $6[3-16](1-106)$ \\
\hline Number of readmissions, median [IQR] (range) & $1[1-2](1-8)$ \\
\hline Emergency department visit & $276(52 \%)$ \\
\hline \multicolumn{2}{|l|}{ Days to the first visit } \\
\hline Mean \pm SD & $84 \pm 92$ \\
\hline Median & $49[14-121](1-361)$ \\
\hline Number of emergency visits, median [IQR] (range) & $2[1-3](1-27)$ \\
\hline Outpatient consultation & $478(90 \%)$ \\
\hline \multicolumn{2}{|l|}{ Days to the first consult } \\
\hline Mean \pm SD & $29 \pm 45$ \\
\hline Median [IQR] (range) & $15[7-321](1-356)$ \\
\hline Number of outpatient visits, median [IQR] (range) & $7[3-13](1-48)$ \\
\hline Day hospital visit & $38(7 \%)$ \\
\hline Laboratory/radiologic examinations & $270(51 \%)$ \\
\hline Psychological/psychiatric service visit & $58(11 \%)$ \\
\hline Rehabilitation outpatient service ${ }^{c}$ & $77(14 \%)$ \\
\hline 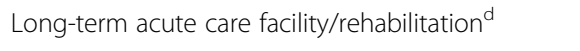 & $16(3 \%)$ \\
\hline 1-yr mortality & $46(9 \%)$ \\
\hline
\end{tabular}

ICU Intensive Care Unit, IQR interquartile range, LOS length of stay, SD standard deviation

${ }^{\text {a }}$ There were 34 patients who were not treated in our facilities after discharge ${ }^{b}$ Refers to the number of the first hospital readmission occurring after an emergency department visit

Includes physiotherapy, nutrition and speech therapist

${ }^{d}$ Patients who were transferred to a long-term acute care or rehabilitation facilities after hospital readmission
Two hundred seventy patients (51\%) visited the service for complementary examinations, and $14 \%$ attended outpatient rehabilitation services, consisting of physiotherapy, nutrition and speech therapy. Fifty-eight patients (11\%) were followed by the psychological/psychiatric service, and $7 \%$ used a day hospital facility. Use of health resources within the first 30 days after hospital discharge was associated with the number of organ failures at ICU discharge (Fig. 4), but not with the number of maximum organ failures during the ICU stay. For 1-year use of health resources, the number of organ failures had minimal impact (Additional file 1: Figure S8).

\section{Discussion}

In our analysis of 690 patients discharged after at least a 72-h stay in the ICU, we found that $27 \%$ of them died within the first year after discharge and that acquired organ failure had an important and non-linear effect on mortality risk. Additionally, a complementary analysis showed an important burden of these patients on a tertiary public health care complex in Brazil.

Mortality after ICU discharge is a well-known phenomenon in the medical literature, and it probably results from the interplay between patient features (e.g., age, baseline performance status) and the severity of the illness [44]. In this analysis, after choosing ICU discharge as the starting point, we demonstrated that long-term prognosis was associated with the degree of organ failure during ICU stays. Moreover, we emphasized that these effects were non-linear and tended to decrease during the year following ICU discharge. We also report that the number of patients with residual organ dysfunction at the time of ICU discharge was significant and that these dysfunctions had an independent long-term prognostic impact. We believe that this study is the first to focus on the long-term outcomes of a subset of critically ill patients with residual organ dysfunction.

The impact of organ failure on post-ICU mortality has been evaluated previously $[1,8,25,26,28,44,45]$, although little is known about residual organ dysfunction. Lone et al. [25] evaluated the impact of organ failure on 5 -year mortality and found that both individual organ failures and the total burden of organ failures (measured by the sum of the worst SOFA score on each domain during the ICU stay) were associated with poorer prognosis, using ICU admission as the starting point for follow-up. The authors performed a landmark analysis, choosing several cut points during the 5-year follow-up period and excluding patients who died before each point [46]. Our study presents a further step of allowing for non-linear and time-varying effects of organ failure and choosing ICU discharge as the starting point. Therefore, we could evaluate an intermediate period during which the patients were at increased risk for mortality (e.g., lower 


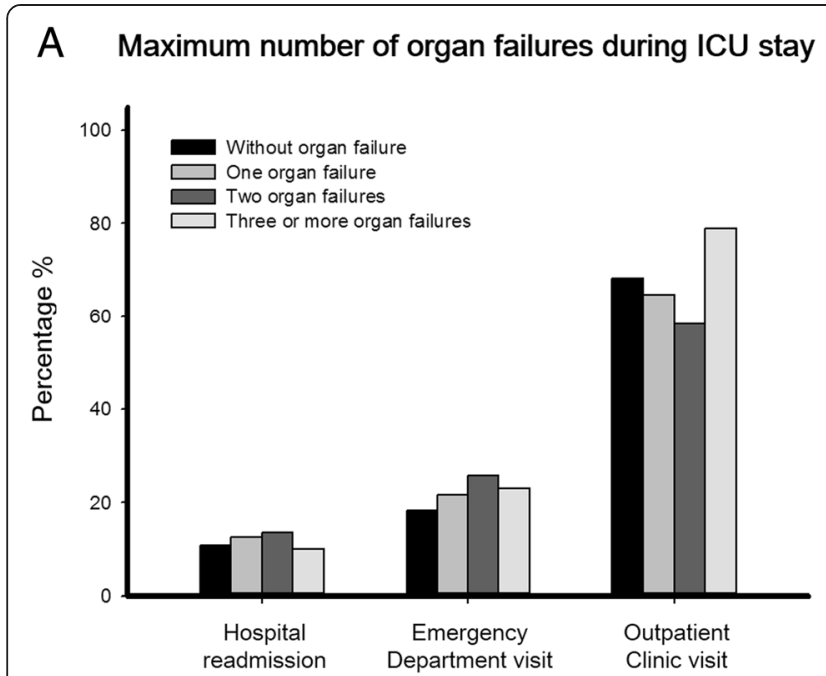

\section{B Number of organ failures at ICU discharge}

Fig. 4 Association between the number of organ failures and health care use in the first 30 days after hospital discharge. (a) Crude associations among the maximum numbers of organ failures [evaluated on the basis of the maximum Sequential Organ Failure Assessment (SOFA) score] during the Intensive Care Unit (ICU) stay. (b) Crude associations among the numbers of organ failures at ICU discharge (evaluated on the basis of the SOFA score at ICU discharge)

risk than during the ICU stay but higher than 90 days later), which is not considered in the landmark method. Using this approach, we found that the impact of organ failure vanishes over time $[14,28]$, which could help in the decision-making process, providing information for a period that is amenable to interventions while the patient is in the hospital.

An important finding of our study was the association between residual organ dysfunction at ICU discharge and 1-year mortality. The association between residual organ dysfunction and worse 1-year survival was clear if at least three residual organ dysfunctions were present (Additional file 1: Figure S5). This finding emphasizes the additive effect of residual organ dysfunction on prognosis. Indeed, a SOFA value of 3, for example, could reflect the presence of one organ failure or three organ systems with a residual degree of dysfunction. Mild degrees might reflect a high burden of residual organ dysfunction owing to increased severity of illness (thereby impacting long-term prognosis), but it might also be consequence of early discharge. The pressure for available beds could play a role in the discharge policy, and interventions in this period have been suggested to decrease the risk of death following ICU discharge [47]. Quantifying residual organ dysfunction at discharge might offer a window of opportunity to identify patients at increased risk for long-term mortality.

Health care use by ICU survivors has been described recently for mechanically ventilated [16, 48], septic [49] and elderly [18] critically ill patients in the United States, for the general population in Canada [19] and for patients in 22 ICUs in the United Kingdom [21]. To the

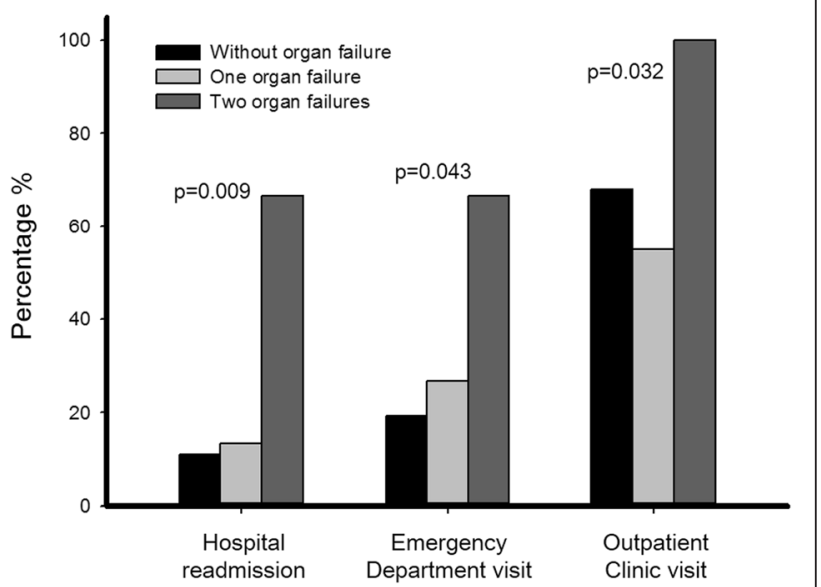

best of our knowledge, the present study is the first of the burden of ICU survivors in a developing country with a national universal public health coverage system. Our results reinforce the special attention required for these patients during the post-hospitalization period. Almost $40 \%$ of patients were rehospitalized, and $52 \%$ visited the emergency department at least once within the first year after discharge, similar to the burden reported in Canada [19]. The number of organ failures was indicative only of early health care use. For long-term health care use, our analysis was underpowered because it was not possible to account for higher use of resources by patients who died earlier [50].

One of the strengths of our study is that it allowed for non-linear and time-varying effects [29]. If one aims to develop interventions that impact long-term prognosis after critical illness, the discharge period might represent the most optimal time to initiate an intervention to modify long-term outcomes. To develop the correct approach after discharge, it is imperative to know how risk factors for long-term mortality behave over time. This behavior can be assessed only if the statistical analysis considers time, which is impossible in common logistic regression and traditional Cox models [14,39].

Nevertheless, this study had some limitations that must be acknowledged. First, it was a single-center analysis; as such, it was subject to local bias, and it had results that were potentially not generalizable. Second, we did not have data on health care use outside our health care complex by these patients; therefore, we could have underestimated their burden. We speculate that this underestimation was low because these patients had a 
high rate of follow-up (93\%) and a significant measured burden after discharge (e.g., $90 \%$ of them presented at an outpatient clinic, with a median of 7 [3-13] outpatient visits in the first year). Third, our sampling was by convenience, and it could have been underpowered. Fourth, organ failure was measured using the SOFA score, which does not measure other signs of organ dysfunction that might be associated with poor prognosis [51-54]. As previously noted by Lone and Walsh [25], the SOFA score can also measure comorbidities (e.g., chronic renal failure on dialysis or cirrhosis), which could influence the results to some degree. Nevertheless, this theory does not explain the reduced impact of the SOFA score over time. Fifth, we did not have data regarding comorbidities such as Charlson or Elixhauser comorbidity index scores, which might have reduced our ability to explore comorbidities more comprehensively. Sixth, we had no data regarding the health care burden during the period before ICU admission [20] and no data from a control group [18]. Finally, we could not represent the private health system in Brazil.

\section{Conclusions}

Both the maximum number of organ failures during ICU stays and the presence of residual organ dysfunction at discharge were associated with poor prognosis in ICU survivors. These associations tended to decrease over time. The use of health care resources was high among ICU survivors, and target actions are necessary to deliver appropriate care for the improvement of the long-term burden of critical illness.

\section{Key messages}

- Organ failure, both in number and intensity, not only represents an acute burden for the critically ill but also impacts 1-year prognosis and resource use after ICU discharge.

- The effects of organ failure on long-term outcomes decrease over time.

- ICU discharge is an important time point in the dynamic process of critical illness, and it represents an opportunity to plan actions and to improve longterm outcomes.

\section{Additional file}

Additional file 1: Complementary figures showing study flowchart, methodology and results.

\section{Abbreviations}

AIC: Akaike Information Criterion; APACHE II: Acute Physiology and Chronic Health Evaluation II; Cl: Confidence interval; CNS: Central nervous system; COPD: Chronic pulmonary obstructive disease; ED: Emergency Department; HR: Hazard ratio; ICU: Intensive Care Unit; IQR: Interquartile range; LOS: Length of stay; PH: Proportional hazards; ROC: Receiver operating characteristic; SD: Standard deviation; SOFA: Sequential Organ Failure Assessment; VIF: Variance inflation factor.

\section{Competing interests}

The authors declare that they have no competing interests.

\section{Authors' contributions}

OTR participated in the conception and design of study, data acquisition, and data analysis and interpretation and also helped to draft the manuscript. FGZ participated in the conception and design of study, data acquisition, and data analysis and interpretation, and also helped to revise the manuscript for important intellectual content. BAMPB carried out data acquisition and interpretation and helped to draft the manuscript. LCPA carried out data acquisition and interpretation and helped to revise the manuscript for important intellectual content. MP participated in the conception and design of study, data acquisition, and data interpretation and helped to revise the manuscript for important intellectual content. All authors read and approved the final manuscript.

\section{Acknowledgments}

We are deeply in debt with Prof. Margaret S. Herridge for her insightful suggestions and review of the manuscript. The study was funded with institutional funds.

\section{Author details}

${ }^{1}$ Intensive Care Unit, Emergency Medicine Discipline, Hospital das Clínicas, University of São Paulo, Rua Dr. Enéas de Carvalho Aguiar, 255, 5th floor, Room 5023, São Paulo 05403-010, Brazil. ${ }^{2}$ Research and Education Institute, Hospital Sirio-Libanes, São Paulo, Brazil.

Received: 19 February 2015 Accepted: 12 June 2015

Published online: 25 June 2015

\section{References}

1. Garland A, Olafson K, Ramsey CD, Yogendran M, Fransoo R. Distinct determinants of long-term and short-term survival in critical illness. Intensive Care Med. 2014;40:1097-105.

2. Brinkman S, Bakhshi-Raiez F, Abu-Hanna A, de Jonge E, de Keizer NF. Determinants of mortality after hospital discharge in ICU patients: literature review and Dutch cohort study. Crit Care Med. 2013;41:1237-51.

3. Capuzzo M, Bianconi M, Contu P, Pavoni V, Gritti G. Survival and quality of life after intensive care. Intensive Care Med. 1996;22:947-53.

4. Azoulay E, Citerio G, Bakker J, Bassetti M, Benoit D, Cecconi M, et al. Year in review in Intensive Care Medicine 2013: II. Sedation, invasive and noninvasive ventilation, airways, ARDS, ECMO, family satisfaction, end-of-life care, organ donation, informed consent, safety, hematological issues in critically ill patients. Intensive Care Med. 2014;40:305-19.

5. Cuthbertson BH, Elders A, Hall S, Taylor J, MacLennan G, McKirdy F, et al. Mortality and quality of life in the five years after severe sepsis. Crit Care. 2013;17:R70.

6. García Lizana F, Manzano Alonso JL, González Santana B, Fuentes Esteban J, Saavedra Santana P. Survival and quality of life of patients with multiple organ failure one year after leaving an intensive care unit. Med Clin (Barc). 2000;114:99-103.

7. Connolly B, Denehy L, Brett S, Elliott D, Hart N. Exercise rehabilitation following hospital discharge in survivors of critical illness: an integrative review. Crit Care. 2012;16:226.

8. Moreno R, Miranda DR, Matos R, Fevereiro T. Mortality after discharge from intensive care: the impact of organ system failure and nursing workload use at discharge. Intensive Care Med. 2001;27:999-1004.

9. Campbell AJ, Cook JA, Adey G, Cuthbertson BH. Predicting death and readmission after intensive care discharge. Br J Anaesth. 2008;100:656-62.

10. Flaatten $H$, Kvåle R. Survival and quality of life 12 years after ICU: a comparison with the general Norwegian population. Intensive Care Med. 2001;27:1005-11.

11. Herridge MS. Long-term outcomes after critical illness: past, present, future. Curr Opin Crit Care. 2007;13:473-5.

12. Contrin LM, Paschoal VD, Beccaria LM, Cesarino CB, Lobo SM. Quality of life of severe sepsis survivors after hospital discharge. Rev Lat Am Enfermagem. 2013;21:795-802. 
13. Haas JS, Teixeira C, Cabral CR, Fleig AH, Freitas AP, Treptow EC, et al. Factors influencing physical functional status in intensive care unit survivors two years after discharge. BMC Anesthesiol. 2013;13:11.

14. Schneider CP, Fertmann J, Geiger S, Wolf H, Biermaier H, Hofner B, et al. Long-term survival after surgical critical illness: the impact of prolonged preceding organ support therapy. Ann Surg. 2010;251:1145-53.

15. Capuzzo M, Ranzani OT. How objective is the observed mortality following critical care? Intensive Care Med. 2013;39:2047-9.

16. Unroe M, Kahn JM, Carson SS, Govert JA, Martinu T, Sathy SJ, et al. One-year trajectories of care and resource utilization for recipients of prolonged mechanical ventilation: a cohort study. Ann Intern Med. 2010;153:167-75

17. Davydow DS, Hough CL, Zatzick D, Katon WJ. Psychiatric symptoms and acute care service utilization over the course of the year following medical-surgical ICU admission: a longitudinal investigation. Crit Care Med. 2014;42:2473-81.

18. Wunsch H, Guerra C, Barnato AE, Angus DC, Li G, Linde-Zwirble WT. Three-year outcomes for Medicare beneficiaries who survive intensive care. JAMA. 2010;303:849-56.

19. Garland A, Olafson K, Ramsey CD, Yogendran M, Fransoo R. A population-based observational study of ICU-related outcomes: with emphasis on post-hospital outcomes. Ann Am Thorac Soc. 2015;12:202-8.

20. Prescott HC, Langa KM, Liu V, Escobar GJ, Iwashyna TJ. Increased 1-year healthcare use in survivors of severe sepsis. Am J Respir Crit Care Med. 2014;190:62-9.

21. Griffiths J, Hatch RA, Bishop J, Morgan K, Jenkinson C, Cuthbertson BH, et al. An exploration of social and economic outcome and associated health-related quality of life after critical illness in general intensive care unit survivors: a 12-month follow-up study. Crit Care. 2013;17:R100.

22. Lone NI, Seretny M, Wild SH, Rowan KM, Murray GD, Walsh TS. Surviving intensive care: a systematic review of healthcare resource use after hospital discharge. Crit Care Med. 2013;41:1832-43.

23. Ranzani OT, Zampieri FG, Forte DN, Azevedo LC, Park M. C-reactive protein/ albumin ratio predicts 90 -day mortality of septic patients. PLoS One. 2013;8:e59321

24. Grander W, Dünser M, Stollenwerk B, Siebert U, Dengg C, Koller B, et al. C-reactive protein levels and post-ICU mortality in nonsurgical intensive care patients. Chest. 2010;138:856-62.

25. Lone $\mathrm{NI}$, Walsh TS. Impact of intensive care unit organ failures on mortality during the five years after a critical illness. Am J Respir Crit Care Med. 2012;186:640-7.

26. Williams TA, Dobb GJ, Finn JC, Knuiman MW, Geelhoed E, Lee KY, et al. Determinants of long-term survival after intensive care. Crit Care Med. 2008:36:1523-30.

27. Hartl WH, Wolf H, Schneider CP, Küchenhoff H, Jauch KW. Acute and long-term survival in chronically critically ill surgical patients: a retrospective observational study. Crit Care. 2007;11:R55

28. Pavon A, Binquet C, Kara F, Martinet O, Ganster F, Navellou JC, et al. Profile of the risk of death after septic shock in the present era: an epidemiological study. Crit Care Med. 2013;41:2600-9.

29. Ranzani OT, Zampieri FG, Park M, Salluh Jl. Long-term mortality after critical care: what is the starting point? Crit Care. 2013;17:191.

30. Flaatten $\mathrm{H}$. Follow-up after intensive care: another role for the intensivist? Acta Anaesthesiol Scand. 2005;49:919-21.

31. Dowdy DW, Needham DM, Mendez-Tellez PA, Herridge MS, Pronovost PJ. Studying outcomes of intensive care unit survivors: the role of the cohort study. Intensive Care Med. 2005;31:914-21.

32. Sakr Y, Vincent JL, Ruokonen E, Pizzamiglio M, Installe E, Reinhart K, et al. Sepsis and organ system failure are major determinants of post-intensive care unit mortality. J Crit Care. 2008;23:475-83.

33. Ranzani OT, Zampieri FG, Taniguchi LU, Forte DN, Azevedo LC, Park M. The effects of discharge to an intermediate care unit after a critical illness: a 5-year cohort study. J Crit Care. 2014;29:230-5.

34. Knaus WA, Draper EA, Wagner DP, Zimmerman JE. APACHE II: a severity of disease classification system. Crit Care Med. 1985;13:818-29.

35. Vincent JL, Moreno R, Takala J, Willatts S, De Mendonça A, Bruining H, et al. The SOFA (Sepsis-related Organ Failure Assessment) score to describe organ dysfunction/failure. Intensive Care Med. 1996;22:707-10.

36. Vincent JL, de Mendonça A, Cantraine F, Moreno R, Takala J, Suter PM, et al. Use of the SOFA score to assess the incidence of organ dysfunction/failure in intensive care units: results of a multicenter, prospective study. Working group on "sepsis-related problems" of the European Society of Intensive Care Medicine. Crit Care Med. 1998;26:1793-800.
37. Moreno R, Vincent JL, Matos R, Mendonça A, Cantraine F, Thijs L, et al. The use of maximum SOFA score to quantify organ dysfunction/failure in intensive care: results of a prospective, multicentre study. Intensive Care Med. 1999:25:686-96.

38. Therneau TM, Grambsch PM. Model survival data: extending the Cox model. New York: Springer; 2000

39. Schneider CP, Fertmann J, Miesen J, Wolf H, Flexeder C, Hofner B, et al. Short-term prognosis of critically ill surgical patients: the impact of duration of invasive organ support therapies. J Crit Care. 2012;27:73-82.

40. Meira-Machado L, Cadarso-Suárez C, Gude F, Araújo A. smoothHR: an R package for pointwise nonparametric estimation of hazard ratio curves of continuous predictors. Comput Math Methods Med. 2013;2013:745742.

41. Harrell Jr FE. Regression modeling strategies: with applications to linear models, logistic regression, and survival analysis. New York: Springer; 2001.

42. Dharmarajan K, Hsieh AF, Lin Z, Bueno H, Ross JS, Horwitz LI, et al. Hospital readmission performance and patterns of readmission: retrospective cohort study of Medicare admissions. BMJ. 2013;347:f6571

43. Ortego A, Gaieski DF, Fuchs BD, Jones T, Halpern SD, Small DS, et al. Hospital-based acute care use in survivors of septic shock. Crit Care Med. 2015;43:729-37.

44. Ho KM, Knuiman M, Finn J, Webb SA. Estimating long-term survival of critically ill patients: the PREDICT model. PLoS One. 2008;3, e3226.

45. Ulvik A, Kvåle R, Wentzel-Larsen T, Flaatten H. Multiple organ failure after trauma affects even long-term survival and functional status. Crit Care. 2007;11:R95.

46. Dafni U. Landmark analysis at the 25-year landmark point. Circ Cardiovasc Qual Outcomes. 2011;4:363-71.

47. Daly K, Beale R, Chang RW. Reduction in mortality after inappropriate early discharge from intensive care unit: logistic regression triage model. BMJ. 2001;322:1274-6

48. Cox CE, Carson SS, Lindquist JH, Olsen MK, Govert JA, Chelluri L, et al. Differences in one-year health outcomes and resource utilization by definition of prolonged mechanical ventilation: a prospective cohort study. Crit Care. 2007;11:R9

49. Winters BD, Eberlein M, Leung J, Needham DM, Pronovost PJ, Sevransky JE. Long-term mortality and quality of life in sepsis: a systematic review. Crit Care Med. 2010;38:1276-83.

50. Wunsch $\mathrm{H}$. Untangling the healthcare use patterns of severe sepsis survivors. Am J Respir Crit Care Med. 2014;190:7-8.

51. Vincent JL, Sakr Y. SOFA so good for predicting long-term outcomes. Resuscitation. 2012;83:537-8.

52. Huang SS, Chen YH, Lu TM, Chen LC, Chen JW, Lin SJ. Application of the Sequential Organ Failure Assessment score for predicting mortality in patients with acute myocardial infarction. Resuscitation. 2012;83:591-5.

53. Cornet AD, Issa Al, van de Loosdrecht AA, Ossenkoppele GJ, van Schijndel RJS, Groeneveld AB. Sequential organ failure predicts mortality of patients with a haematological malignancy needing intensive care. Eur J Haematol. 2005;74:511-6.

54. de Mendonça A, Vincent $J$, Suter PM, Moreno R, Dearden NM, Antonelli M, et al. Acute renal failure in the ICU: risk factors and outcome evaluated by the SOFA score. Intensive Care Med. 2000;26:915-21.

\section{Submit your next manuscript to BioMed Central and take full advantage of:}

- Convenient online submission

- Thorough peer review

- No space constraints or color figure charges

- Immediate publication on acceptance

- Inclusion in PubMed, CAS, Scopus and Google Scholar

- Research which is freely available for redistribution 